Portland State University

PDXScholar

University Honors Theses

University Honors College

2014

Cellular Automata as Cellular Spaces

Max Orhai

Portland State University

Follow this and additional works at: https://pdxscholar.library.pdx.edu/honorstheses

Let us know how access to this document benefits you.

Recommended Citation

Orhai, Max, "Cellular Automata as Cellular Spaces" (2014). University Honors Theses. Paper 87.

https://doi.org/10.15760/honors.90

This Thesis is brought to you for free and open access. It has been accepted for inclusion in University Honors Theses by an authorized administrator of PDXScholar. Please contact us if we can make this document more accessible: pdxscholar@pdx.edu. 


\title{
Cellular Automata as Cellular Spaces
}

\author{
by \\ Max Orhai
}

An undergraduate honors thesis submitted in partial fulfillment of the

\author{
requirements for the degree of \\ Bachelor of Science \\ in \\ University Honors \\ and \\ Mathematics
}

Thesis Adviser

M. Paul Latiolais

Portland State University 


\title{
CELLULAR AUTOMATA AS CELLULAR SPACES
}

\author{
MAX ORHAI
}

\begin{abstract}
The topology of an abstract cellular complex is developed from the idea of a locally finite space consisting of countably many elements, and can provide a coordinatefree definition of both the geometric structure and discrete dynamics of cellular automata.
\end{abstract}

\section{INTRODUCTION}

Cellular automata (or $C A$ ) are a class of abstract models of computation, typically distinguished by their discrete geometric structure and localized state transformation rules. Originally described independently by Stanislaw Ulam and John von Neumann in the early 1940s, they have been applied as scientific modeling tools in fields as diverse as theoretical biology, urban studies, and physics. Specific configurations of cellular automata can be considered as principled models of distributed algorithms, a topic of increasing interest in both theoretical and applied computer science as computing systems have grown in scale to approach physical limits on centralized coordination.

Cellular automata are discrete dynamical systems, typically defined on integer lattices, or sometimes on graphs. In this paper, I will characterize cellular automata topologically, using the concept of an abstract cell complex together with basic concepts of automata theory. The practical advantages of using this more elaborate topological structure are several. In general, it allows us access to a mature and well-developed theory in which we can reason rigorously about spatial relationships between the abstract elements of distributed computing systems as well as the physical space-time of their concrete implementations. In particular, I will prove that the digital geometry I present satisfies the classical axioms for topological spaces, including the TO separation axiom. I will investigate the relationship of a particular kind of this geometry to the familiar two-dimensional Euclidean plane. Finally, I will show how this decentralized, coordinate-free topological characterization can be used to define two specific well-known cellular automata in a way that enables mathematical reasoning about the spatial as well as computational properties of these systems. I hope to demonstrate to the satisfaction of the reader how the topology of cellular complexes can be applied to enhance both the theory and practice of spatially distributed computing.

Date: June 7, 2014.

Prepared with gratitude for the advice and inspiration of Professor M. Paul Latiolais, the faculty of the Mathematics and Computer Science departments, and my fellow students at Portland State University. 


\section{TOPOLOGY OF LOCALLY FINITE SPACES}

In this section I will present an axiomatic development of the structure of an abstract locally finite space, using some elementary notions from Zermelo-Fraenkel set theory in their standard notations. These include unordered collections or sets which I will generally denote with capital letters and curly brackets $A=\{a, b, c\}$; the empty set $\emptyset$; inclusion or membership of distinct elements $a \in A$; subsets $B \subseteq A$, meaning that $b \in B$ implies $b \in A$; unions of sets $A \cup B=\{x: x \in A$ or $x \in B\}$; intersections of sets $A \cap B=\{y: y \in A$ and $y \in B\}$; set difference $A-B=\{a \in A: a \notin B\}$ and the power set $\mathcal{P}(A)=\{B: B \subset A\}$. I will use lowercase Greek letters to denote functions $\alpha: A \rightarrow B$, which we can consider as sets of ordered pairs $(a, b)$ assigning to each $a \in A$ some unique $b \in B ; A$ is called the domain and $B$ the range of $\alpha$. Sets of ordered pairs $\Gamma \subseteq\left\{\left(a, a^{\prime}\right): a, a^{\prime} \in A\right\}$, or relations, I will denote with Greek capitals. A relation $\Gamma$ on a set $A$ is called reflexive if $a \in A \Rightarrow(a, a) \in \Gamma$, symmetric if $(a, b) \in \Gamma \Rightarrow(b, a) \in \Gamma$, and transitive if $(a, b),(b, c) \in \Gamma \Rightarrow(a, c) \in \Gamma$.

Axioms and definitions in this section will be used as a spatial framework for cellular automata throughout the rest of the paper, where we will see several different examples of these structures. The axiom scheme and much of its terminology is due to Vladimir Kovalevsky [3]. It is a particular instance of an abstract cell complex structure, originally proposed in 1862 by J.B. Listing — who first introduced the term "topology" — and is most often applied to digital image analysis: see for example Klette and Rosenfeld [4].

Definition. A locally finite space is simply a nonempty set $S$ of elements $e$, together with a function $\tau: S \rightarrow \mathcal{P}(\mathcal{P}(S)$ ) which assigns to each $e \in S$ a collection of subsets of $S$ called neighborhoods, at least one of which, say $T$, is finite. Formally, $\forall e \in S \exists T \in \tau(e):|T| \in \mathbb{N}$. Any subset $T \subset S$ is called a subspace of $S$.

For example, consider a set $V$ and a relation $\Gamma \subseteq\{(u, v): u, v \in V\}$. These together constitute a directed graph, where elements of $V$ are vertices, and elements of $\Gamma$ are directed edges from vertex $u$ to $v$. (If $\Gamma$ is symmetric, then we can regard its elements as unordered pairs $\{u, v\}$, and the entire structure as an undirected graph.) Now if we define the function $\tau(u)=\{\{v\}:(u, v) \in \Gamma\}$, our graph is a locally finite space, since $|\{v\}|=1 \in \mathbb{N}$. We will see later in this section how with only a little more structure we can equally characterize a graph as a one-dimensional abstract cell complex.

As another example, we can define a locally finite space on the integers $\mathbb{Z}$ with the function $\tau$ which assigns to each $n \in \mathbb{Z}$ the set of sets of consecutive integers which include $n:\{\{a, \ldots, n, \ldots, b\}: a \leqslant n \leqslant b\}$. Then any particular integer, say 0 , has neighborhoods $\tau(0)=\{\{0\},\{-1,0\},\{0,1\},\{-1,0,1\}, \ldots\}$. Although the set $\tau(n)$ is infinite for every $n$, and the infinite set $\mathbb{Z} \in \tau(n)$ is a specific neighborhood of every $n$, so is the singleton set $\{n\} \in \tau(n)$. Since $|\{n\}|=1 \in \mathbb{N}$, the definition's condition is fulfilled.

To avoid confusion, we will not call the space elements $e \in S$ 'points,' since they do not generally possess the homogeneity one might expect from classical 'point-set' topology. However, as we shall see in Subsection 2.2, the structures with which we are concerned are nonetheless legitimate topological spaces satisfying the classical axioms. 
The local-finiteness condition is necessary, although not sufficient, to assure that the dynamic aspects of the automata which we will see in Sections 3 and 4 remain computable. All infinite sets present in the definitions that follow are countable by construction, obviating the need for any additional axioms in our set theory. In what follows, I will make use of the integers $\mathbb{Z}$, including the non-negative integers $\mathbb{N}$, and the associated notion of a vector or finite sequence of elements in a set $A$ ordered by an integer index $\left(a_{0}, a_{1}, \ldots, a_{i}, \ldots, a_{k}\right): a_{i} \in A, 0 \leqslant i \leqslant k \in \mathbb{N}$, taking these structures as well defined. Later in this section we will see how the AC complexes defined below can be interpreted or embedded as geometric structures in the familiar Euclidean spaces $\mathbb{E}^{n}$. However, I will take care to show that the characterization of a cellular automaton which I develop here does not depend on the topology of $\mathbb{E}$, or on any pre-existing global coordinate system, but rather develops an abstract theory of spatial relations from the "bottom-up" point of view of individual elements and their neighborhoods. This perspective is important in the design of large-scale distributed computing systems, where global information, including absolute addressing, may be unavailable at the component level.

2.1. ALF spaces and AC complexes. We can restrict the definition of a locally finite space $S$ in such a way as to ensure that the resulting structure (an ALF space) has welldefined notions of connectedness, boundary, and separability, and satisfies the classical axioms for topological spaces. Following Kovalevsky, I will do this by introducing four axioms for ALF spaces, together with some associated definitions, and derive a few important properties of these structures. The additional feature of a dimension function will further restrict ALF spaces to the class of AC complexes, which will provide the digital geometric framework for the full definition of a cellular automaton which I develop in Section 4. In Subsection 2.2, I will show how the classical axioms of topology can be derived from the ALF axioms.

ALF Axiom 1. Each element $e$ of space $S$ is a member of each of its neighborhoods, and the intersection of any two such neighborhoods is also a neighborhood of $e$. Formally, $T \in \tau(e) \Leftrightarrow e \in T$ and $T, T^{\prime} \in \tau(e) \Leftrightarrow T \cap T^{\prime} \in \tau(e)$.

Definition. The smallest neighborhood function $\sigma: S \rightarrow \mathcal{P}(S)$ assigns to an element $e \in S$ the intersection of all neighborhoods of $e$. The neighborhood $\sigma(e)$ always exists and is finite and nonempty as a direct consequence of ALF Axiom 1 and the definition of a locally finite space. Formally, $\sigma(e)=\left\{e^{\prime} \in S: T \in \tau(e) \Rightarrow e^{\prime} \in T\right\}$.

ALF Axiom 2. Some elements $e$ of space $S$ have a smallest neighborhood $\sigma(e)$ containing more than one element; $\exists e \in S:|\sigma(e)|>1$.

Definitions. In the context of a locally finite space $S$, the boundary $\beta:(\mathcal{P}(S)-\{\emptyset\}) \rightarrow$ $\mathcal{P}(S)$ assigns to each nonempty subspace $T \subset S$ the subspace of $S$ containing exactly the elements $e$ whose smallest neighborhoods include elements in both $T$ and its complement $S-T$. Formally, $\beta(T)=\{e \in S: \sigma(e) \cap S \neq \emptyset$ and $\sigma(e) \cap(S-T) \neq \emptyset\}$. A boundary $\beta(T)$ is called thick if it contains any opponent pairs $\{a, b\}$ such that $a \in \sigma(b), b \in \sigma(a), a \in T$, and $b \in S-T$. Otherwise, $\beta(T)$ is thin. 
ALF Axiom 3. The boundary $\beta(T)$ of a nonempty subspace $T \subseteq S$ is thin.

ALF Axiom 4. The boundary of $T$ is its own boundary: $\beta(\beta(T))=\beta(T)$.

It will be helpful to define a small vocabulary of supporting notions. First, the direct neighbor relation on $S$ is a set of ordered pairs of elements $\Delta=\{(b, a): b \in \sigma(a)\}$. If $(b, a) \in \Delta$ and $a \neq b$ then we say $b$ is bounded by $a$ (or $a$ bounds $b$ ). The inverse relation $\Phi=\{(a, b): b \in \sigma(a)\}$ is called the facial relation; $a$ is a face of $b$ if $b \in \sigma(a)$, and a proper face of $b$ if also $a \neq b$. In other words, the smallest (or direct) neighborhood of an element is the set of all the elements which it bounds - or equivalently, of which it is a face. We say $a$ is incident (or directly connected) to $b$ in case $(a, b) \in \Psi=\Delta \cup \Phi$; this incidence relation $\Psi$ is by definition symmetric. A path in a space $S$ is subspace which can be written as a finite sequence of directly connected elements: $\left(e_{0}, \ldots, e_{i}, \ldots, e_{k}\right): e_{i} \in$ $S,\left(e_{i-1}, e_{i}\right) \in \Psi \forall 0<i \leqslant k \in \mathbb{N}$. Any two elements $a, b \in S$ are connected if and only if a path exists with $a=e_{0}$ and $b=e_{k}$. A bounding path is a path in which $e_{i-1}$ bounds $e_{i}$ for all $0<i \leqslant k$.

Theorem 1. The antisymmetry of $\Delta$ is equivalent to ALF Axiom 3.

Proof. If Axiom 3 holds, then the boundary of subspace $T$ is thin, with no opponent pairs of mutually bounding elements $a \neq b$ such that both $(a, b),(b, a) \in \Delta$. Thus $\Delta$ is antisymmetric. Conversely, if $\Delta$ is not antisymmetric, then there is a distinct pair of elements $a, b \in T$ such that $a \in \sigma(b)$ and $b \in \sigma(a)$. Then both $a, b \in \beta(\{a\})$, since $\sigma(a)$ contains both an element of $\{a\}$ and the element $b \in S-\{a\}$. Thus $a, b$ are an opponent pair, $\{a\} \subset S$, and $\beta(\{a\})$ is thick, not thin.

Theorem 2. The transitivity of $\Delta$ is equivalent to ALF Axiom 4.

Proof. First I will show that if $\Delta$ is transitive, then $\beta(\beta(T))=\beta(T)$, and Axiom 4 holds. Let $a \in \beta(T)$, to show that $a \in \beta(\beta(T))$. By Axiom 1, $a \in \sigma(a)$, so $\sigma(a) \cap \beta(T) \neq \emptyset$. Since $\sigma(a)$ is finite, direct neighbor transitivity implies that $\sigma(a)$ must contain both a minimum element bounded by no other elements in $S$ and a maximum element $m$ which bounds no other elements in $S$. In particular, $m \notin \sigma(a)$, so $m \in S-\beta(T)$, and thereby $\sigma(a) \cap(S-\beta(T)) \neq \emptyset$. Thus $a \in \beta(\beta(T))$. Now let $b \in \beta(\beta(T))$, to show that $b \in \beta(T)$. We have $\sigma(b) \cap \beta(T) \neq \emptyset \neq(S-\beta(T)) \cap \sigma(b)$, so there is some $c \in \sigma(b) \cap \beta(T)$, and the transitivity of $\Delta$ tells us that any $d \in \sigma(c)$ is also in $\sigma(b)$. So $\sigma(c) \subseteq \sigma(b)$, and consequently $\sigma(b)$ intersects both $T$ and $S-T$. Thus $b \in \beta(T)$, fulfilling Axiom 4 .

Conversely, we'll see that Axiom 4 implies the transitivity of $\Delta$, by the contrapositive. Assume $\Delta$ is not transitive, so that there are distinct elements $a, b, c \in S$ with $b \in \sigma(a), c \in$ $\sigma(b)$, but $c \notin \sigma(a)$. Then $a \notin \beta(\{c\})$, since $\sigma(a) \cap\{c\}=\emptyset$. However, $a \in \beta(\beta(\{c\}))$, and since $b$ bounds $c$, moreover $b \in \beta(\{c\})$. So both $\sigma(a) \cap \beta(\{c\})$ and $\sigma(a) \cap(S-\beta(\{c\}))$ are nonempty, contradicting Axiom 4.

By ALF Axiom 1 the direct neighbor and facial relations $\Delta$ and $\Phi$ are reflexive, and by definition the bounding and proper-face relations $\Delta^{\prime}$ and $\Phi^{\prime}$ are irreflexive. Theorems 1 and 2 show that $\Delta$ and $\Phi$ are both antisymmetric and transitive. Thus all four of these relations are partial orders, with $\Delta^{\prime}$ and $\Phi^{\prime}$ strict. I'll write $a<b$ when $a$ bounds $b$, 
and $a \leqslant b$ when $a$ is a face of $b$. The connection relation, being reflexive, symmetric and transitive by definition, is an equivalence relation: I will write $a \approx b$ when $a$ and $b$ are connected, and $a \sim b$ when incident, i.e. directly connected.

2.2. Topology of ALF spaces. Classically, a topology on a set $X$ is a collection of subsets of $X$ called open sets, which satisfy the following axioms:

Classical Axiom 1. Both $X$ and $\emptyset$ are open.

Classical Axiom 2. Any union of open sets is open.

Classical Axiom 3. Any intersection of a finite number of open sets is open.

There are several additional standard topological axioms specifying ways in which nonidentical elements can be 'seperated' or distinguished from each other in terms of open sets. Of these, the only one in common use which is applicable to finite sets is the following:

Separation Axiom. T0: If elements $x, y \in X$ and $x \neq y$, then the topology on $X$ includes an open set containing $x$ but not $y$, or one containing $y$ but not $x$.

Definition. The open sets of an ALF space $S$ are the subspaces $O \subseteq S$ that do not contain any part of their boundary: $O \cap \beta(O)=\emptyset$. Closed sets $C \subseteq S$ are those which contain their entire boundary: $\beta(C) \subseteq C$.

Theorem 3. An ALF space $S$ is a T0 topological space.

Proof. First I'll show that a subspace $T$ is open in $S$ if and only if it contains the smallest neighborhood $\sigma(e)$ of each of its elements $e \in T$. It follows that an ALF space is a topological space satisfying all the classical axioms; this result implies that $\sigma(e)$ is open for every $e \in S$. Finally, we'll see that $S$ satisfies Separation Axiom T0.

Suppose that $T$ is open, $e \in T, e^{\prime} \in \sigma(e)$, and $e^{\prime} \in S-T$. Then we'd have $e \in \beta(T)$, contradicting T's openness. So if $T$ is open, it contains all $\sigma(e)$ for each $e \in T$. Now suppose that $T$ contains each of its elements' smallest neighborhoods, but is not open. Then there must be some element $e^{\prime} \in T$ that is also in $\beta(T)$. But by definition the boundary $\beta(T) \cap(S-T) \neq \emptyset$, so $T$ does not contain $\sigma\left(e^{\prime}\right)$, another contradiction. Thus $T$ is open in $S$ iff $e \in T$ implies $\sigma(e) \subseteq T$.

The empty ALF subspace $\emptyset$, having no elements and thus no smallest neighborhoods, is trivially open in $S$. The boundary of the entire space $\beta(S)=\emptyset$, since $S-S=\emptyset$. So $S$ contains no elements of $\beta(S)$ and is therefore open in itself. Since $S$ is locally finite, its open sets are countable, starting from the smallest neighborhood of any element and proceeding outward. Let each open set $O_{i}$ be indexed by a non-negative integer $i \in \mathbb{N}$. Consider an arbitrary union of open sets $U=O_{i_{0}} \cup O_{i_{1}} \cup \ldots$, with each $i_{k} \in \mathbb{N} ; U$ may be finite or infinite. So if $e \in U$, then $\sigma(e) \subseteq O_{i}$ for some $i \in \mathbb{N}$ and thus $\sigma(e) \subseteq U$, so $U$ is open in $S$. Now consider an element of an arbitrary countable intersection $e \in I=O_{i_{0}} \cap O_{i_{1}} \cap \ldots$ belonging to each open set $O_{i}$. Since $\sigma(e) \subseteq O_{i}$ for all $i, \sigma(e) \subseteq I$, and thus $I$ is open in $S$. This stronger version of the third classical axiom is typical of Alexandroff spaces.

We will now see that smallest neighborhood $\sigma(e)$ of any element $e \in S$ is open, implying that $S$ is T0. Suppose to the contrary that $\sigma(e)$ was not open: then it must contain an 
element $b \in \beta(\sigma(a))$, so there must be an element $c \in \sigma(b)$ yet $c \notin \sigma(a)$. This contradicts the transitivity of the neighbor relation $\Delta$ shown above: consequently $\sigma(e)$ is open in $S$. Now consider any two elements $a, b \in S$. If they are not incident, then both $a \notin \sigma(b)$ and $b \notin \sigma(a)$, satisfying T0. On the other hand, if they are incident, then either $a \in \sigma(b)$ or $b \in \sigma(a)$ : both conditions cannot hold because of the antisymmetry of $\Delta$. So one of $a \notin \sigma(b), b \notin \sigma(a)$ is true, in accordance with T0.

\subsection{Abstract cell complexes and digital geometry.}

Definition. An abstract cell complex or AC complex is an ALF space $C$ equipped with a function $\delta: C \rightarrow \mathbb{N}$ assigning each element $c \in C$ a dimension, which is the smallest non-negative integer $\delta(c)$ such that if $c^{\prime} \in \sigma(c)$ (so $c \leqslant c^{\prime}$ ) then $\delta(c) \leqslant \delta\left(c^{\prime}\right)$.

The elements of an AC complex are called cells, and typically prefixed with their dimension. For reasons that will become clear later in this section, I will use the suggestive terms dot, link, tile, and block for 0, 1, 2, and 3-cells respectively. A subset of a cell complex is called a subcomplex. The dimension $\delta(c)$ of a cell $c$ is defined as one less than the number of cells in the longest bounding path from any cell $a_{0} \in C$ to $c$. Formally, $\delta(c)=\max \left\{k: a_{0}<\cdots<a_{i}<\cdots<a_{k}<c, 0 \leqslant i \leqslant k, a_{i} \in C\right\}$. The dimension $\delta(C)$ of an entire AC complex $C$ is the highest dimension of any of its constituent cells; $\delta(C)=\max \{\delta(c): c \in C\}$. The cells with highest dimension in an AC complex have smallest neighborhoods containing only themselves, so ALF Axiom 2 rules out 0-dimensional complexes, classically known as discrete spaces. Two AC complexes $C$ and $C^{\prime}$ are called isomorphic if and only if there is a one-to-one function from the $d$-cells of $C$ onto the $d$-cells of $C^{\prime}$ for each non-negative integer $d \leqslant \delta(C)=\delta\left(C^{\prime}\right)$.

One particular family of AC complexes is well known in algebraic topology, and has the advantage of an easy definition: an abstract simplicial complex $S$ is a collection of nonempty finite sets (the cells of $S$, called simplexes), such that if simplex $A \in S$ and nonempty $B \subset A$, then also $B \in S$. For simplicial complexes, in other words, the facial relation between cells is just the subset relation, and the dimension $\delta(A)=|\{A\}|-1$. We can give a geometric interpretation to a simplex by embedding it in a Euclidean space of its dimension: 0-simplices (dots) become points, 1-simplices (links) become line segments between the points, 2 -simplices (tiles) become triangles spanning the area between the line segments, 3-simplices become tetrahedra enclosing the volume bounded by the triangles, and so on. The details of this embedding are straightforward, but not especially relevant to this project. However, the geometric intuition that they suggest is useful, especially if made a little less rigid. In what follows, I will picture links as arcs between dots, tiles as polygons whose edges are links, and blocks as polyhedra whose faces are tiles. This is a reasonable geometric interpretation of the combinatorial structure of an AC complex, since we can map a topological open $n$-ball to each $n$-cell, which is always bounded by a finite number of cells, each having dimension less than $n$. In fact, the combinatorial structure of AC complexes is structurally equivalent to a particular family of $C W$ complexes, standard combinatorial topological spaces. The relation between $\mathrm{AC}$ and $\mathrm{CW}$ complexes is essentially very similar to that between abstract and geometric simplicial complexes: the geometric interpret the 
countable cellular structures in terms of uncountable point-sets in a Euclidean topology. But while $n$-cells in an AC complex must be bounded by $(n-1)$-cells, the $n$-cells of a general CW complex need only be bounded by cells with dimension less than $n$.

The simplicial structure also suggests a helpful way of writing down the structure of those AC complexes in which each cell is uniquely determined by its proper faces and thus ultimately by its bounding dots, as is the case in Figure 1a, and will be for most of the examples we'll see in the following sections. For these complexes I'll denote dots with lowercase letters $a, a^{\prime}, b, c$; links as pairs of dots $a b$ meaning $\{a, b\}$; and tiles as cycles of dots, each bounding exactly two of the links bounding the tile, for example abcd meaning the 'square' tile with boundary $\{a, a b, b, b c, c, c d, d, d a\}$; and similarly for blocks, which have boundaries in which each link bounds exactly two tiles, as in the 'square-based pyramid' oabcd with boundary $\{o, a, b, c, d, o a, o b, o c, o d, a b, b c, c d, d a, o a b, o b c, o c d, o d a, a b c d\}$. The dimension of a cell written in this way should be clear from context. However, the notational convenience of this decomposition should not be mistaken for a distinguishing characteristic of AC complexes. For example the 'figure 8,' having two distinct links both bounded by a single dot, is a valid AC complex. So is the 'bigon,' with two dots, two links, and one tile. We could even interpret a finite Von Neumann inclusion ordinal, say $3 \equiv\{\emptyset,\{\emptyset\},\{\emptyset,\{\emptyset\}\}\}$, as a 3-cell in a 'monohedral' complex with membership as the facial relation, by calling $\emptyset$ a 0-cell. Geometric interpretations of these 'exotic' complexes are pictured in Figure 1b. For our purposes we can safely ignore them.

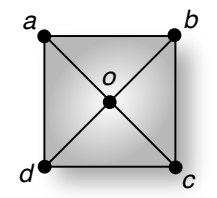

(A) A pyramidal block

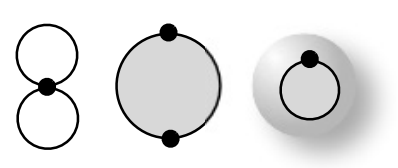

(B) Exotic complexes

Figure 1. Some small AC complexes

We'll be mostly concerned with uniform complexes which can fill Euclidean spaces under our geometric interpretation, since these are the traditional spatial configurations of most cellular automata. The reasons for this focus are essentially computational in nature: we only want to consider objects which admit a finite description, but we need a countably infinite structure in order to be capable of universal (Turing-complete) computation. For infinite complexes, these requirements imply some form of regularity: repetition of a uniform motif is a simple way to achieve this end. The 2-dimensional complexes shown in Figure 2 are prototypical examples. We'll see more of these spaces in Section 4, but first I will give some formal definitions for these tiling complexes in terms of properties of their constituent cells and neighborhoods, rather than a global coordinate system. I will then show that they have the topological characteristics we would expect of a geometric plane.

The cell consolidation which extracts the square and hexagonal tilings from the simplicial one is an operation $\kappa(C, x, z)$ on an $\mathrm{AC}$ complex $C$. It takes two cells $x$ and $z$, both 


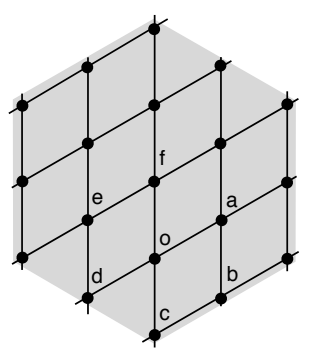

(A) Square

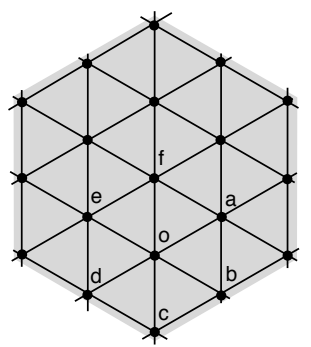

(B) Simplicial

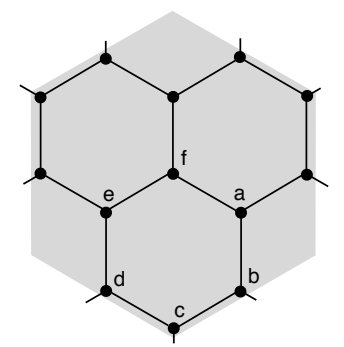

(c) Hexagonal

Figure 2. Neighborhoods in some uniform tiling complexes

of dimension $k$ and having a non-empty exclusive common boundary $Y=\{y \in C: \sigma(y)=$ $\{x, z\}\}$, a subcomplex composed of all cells in $C$ which bound only $x$ and $z$. It produces a new complex $C^{\prime}=\left\{y^{\prime}\right\} \cup C-\{x, z\}-Y$ in which all three of these are replaced by a new $k$-cell $y^{\prime}$ with boundary $\beta\left(y^{\prime}\right)=(\beta(x) \cup \beta(z))-Y$. For example, the simplicial tiles $o a b$ and $o b c$ in Figure $2 \mathrm{~b}$, with exclusive common boundary $\{o b\}$, have been consolidated into the square tile $o a b c$ in Figure 2a. But to produce the hexagonal tile abcdef of Figure $2 \mathrm{c}$ from the six simplicial cells labeled in Figure $2 \mathrm{~b}$ requires six consolidations, the last of which would involve an exclusive common boundary consisting of the dot $o$ and two of its bounded links.

We need to introduce one more notion in order to proceed. Recall that a path in an ALF space is a subspace which can be written as a finite sequence of elements, each of which is directly connected to the previous. Now a loop in an AC complex $C$ is a 1-dimensional path $L=\left(e_{0} \sim e_{0} e_{1} \sim e_{1} \sim \cdots \sim e_{i-1} \sim e_{i-1} e_{i} \sim e_{i} \sim \cdots \sim e_{k} \sim e_{k} e_{0} \sim e_{0}\right)$ where $0<i \leqslant k$ and each $e_{i}$ is a dot. If all $e_{i}$ are distinct from each other, then we'll say $L$ is a proper loop. Notice that the boundary of any tile is a proper loop.

Definition. A uniform tiling complex $T$ is an infinite 2-dimensional $\mathrm{AC}$ complex such that:

(1) Any two cells in $T$ are connected.

(2) Any two $k$-cells in $T$ have isomorphic smallest neighborhoods.

(3) Any proper loop in $T$ is the boundary of a subcomplex which can be consolidated into a single tile.

The surface of a regular polyhedron would fulfill these three criteria, but it's finite. An infinite collection of disjoint polyhedra wouldn't meet the first condition. The third condition rules out infinite cylinders and Moebius bands, or any other spaces with holes: it is essentially the requirement that $T$ be a simply connected space in which all loops are contractible.

Theorem 4. A uniform tiling complex $U$ in which each link bounds more than one tile can be interpreted as a CW complex that is homeomorphic to the Euclidean plane. 
Proof. Consider any dot $o$ in $U$. Since $U$ is an 2-dimensional complex and $\sigma(o)$ is isomorphic to $\sigma\left(o^{\prime}\right)$ for any other $\operatorname{dot} o^{\prime} \in U$, there must be both a link $l$ and a tile $t$ in $\sigma(o)$. Since $U$ is infinite and each of its cells must be bounded by dots, there are infinitely many dots in $U$. Since a dot must have a finite smallest neighborhood and any two dots are connected, there must be infinitely many links. If there were only one tile in $U$, it would have an infinite boundary. But since there are at least two tiles, there must be infinitely many, because they must be in the smallest neighborhood of a link, and thus also of a dot. Could $U$ be an infinite chain of closed bigons, joined by single dots? No, since any link $l$ must bound at least two tiles. In fact, it must bound exactly two, since if $l$ were to bound three tiles, then any two of the three could not be consolidated. Since any two adjacent tiles $t, t^{\prime}$ can be consolidated into a tile whose boundary is in $U$, the result follows from the contractibility of a CW 2-cell.

Topologically, $U$ is connected but not compact: it is an open surface with a trivial fundamental group. Since it is has infinite extent and no holes, it's reasonable for us to think of it and draw it as a subdivided plane. But its geometric interpretations via embeddings into Euclidean spaces need not be geometrically homogenous. Consider, for example, the uniform tiling complex shown in Figure 3.

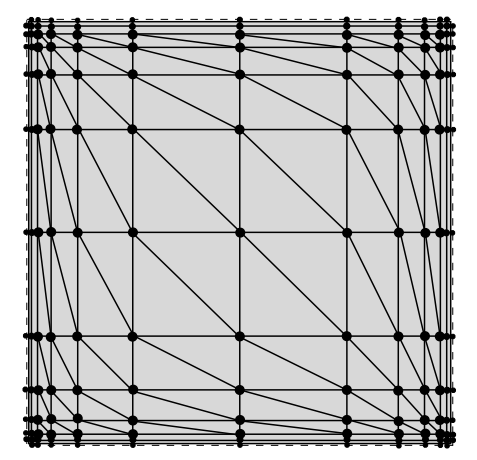

Figure 3. An infinite simplicial tiling embedded in a unit square

I've interpreted the dots in this simplicial tiling complex as the Euclidean points $\{(x, y)$ : $\left.x, y \in\left\{ \pm\left(2^{n}-1\right) / 2^{n+1}: n \in \mathbb{N}\right\}\right\}$. Note that the tiling doesn't contain its limit, the boundary with corners at $\pm 1 / 2$, which is exactly the unit square. Topologically, this is a tiling of an open disc, which is homeomorphic to the entire plane. The topological uniformity of the tiling need not correspond to Euclidean geometric regularities: in particular, the simplices are not equilateral triangles. Many other such embeddings are equally possible, but we will prefer to draw tilings and similar structures as regularly as possible. In what follows, I will use the term "geometry" to refer to the topological structure of a tiling or similar AC complex, rather than the Euclidean geometry of its possible embeddings. 


\section{Automata as complexes}

In the previous section, I hope to have shown to your satisfaction that it's possible to describe the regular grids with which cellular automata are usually associated as topological spaces with their own coordinate-free digital geometry. In this section I will show that the dynamics of cellular automata can also be described in substantially the same language. In the following section we will put the geometric and dynamical pieces together to sketch out a theory of spatially distributed computation.

Recall that a graph is a locally finite space, with vertices as elements. If we regard the vertices as 0-cells or 'dots,' and graph edges as 1-cells or 'link' elements in the space (rather than just neighborhoods), then a graph is indeed an $\mathrm{AC}$ complex of dimension 1. For our present purposes, it's useful to give a direction or orientation to the links, distinguishing between $a b$ and $b a$, both of which connect dots $a$ and $b$ but in different directions. I'll draw these oriented links as arrows, and sometimes describe them with temporal metaphors, since they represent individual 'steps' in a computation. Our existing notion of a path must be slightly extended, in the obvious way, to that of a directed path which respects link orientations, and which may be infinite. Since $a \approx b$ means only that elements $a$ and $b$ are path-connected, and not that a directed path exists between them in either direction, I'll use the notation $a \rightsquigarrow b$ to indicate the existence of a directed path from $a$ to $b$. The orientation of a link can be thought of as a kind of decoration or labeling of the cell. We will label dots and other cells too, with symbols called states. Individual state symbols are drawn from a finite set, although they may form potentially infinite combinations. We will often refer to neighborhood states or even global state configurations of an entire automaton complex at a particular 'step' or moment in time: these labelings are in fact functions from the cells of a subcomplex into the set of possible states. If we give each dot in a directed graph a unique state label, then the graph is called a state transition system or an abstract rewriting system, and we'll often refer to dots themselves as states. If the system is finite, then it's usually called a finite automaton.

Finite automata typically distinguish certain states as 'initial' and 'terminal,' and may also give additional labels to their links (often called 'transitions'). They are usually thought of as recognizing or generating a regular language of symbol sequences, but that perspective is not essential for our purposes. The important distinction here is between deterministic automata, in which there is at most one outbound link from each dot, and nondeterministic automata, where dots may have multiple outbound links. Deterministic automata are sometimes called discrete dynamical systems. Nondeterministic finite automata (NFA) are generally simpler descriptions of the behavior of a particular system, but can always be converted into deterministic finite automata (DFA) whose states are sets of NFA states. We'll see an example of this kind of conversion in the following section.

A directed path through a finite automaton must either end at some dot or infinitely revisit the same dots: in a DFA, the only infinite paths are loops. In typical cellular automata, there are no genuine terminal states, although there may be fixed points, which are dots linked only to themselves. Initial states may be specified, and for a deterministic system they suffice to specify an entire infinite path, called the system's history or evolution. 
An 'unreachable' initial state $S$, for which there is no other state $S^{\prime}$ such that $S^{\prime} \rightsquigarrow S$, is called (poetically enough) an Eden state.

A directed labeled 1-dimensional AC complex which describes the dynamics (although not the geometry) of a cellular automaton is called a rule. It is specified by associating states with certain cells and subcomplexes of the CA's geometry, and must be finite. We can also attribute a state to each possible configuration of geometric cell states in the entire complex. For cellular automata that have finite geometries, we can construct a 'global' DFA for the entire system, although it may have an impractically large number of states. Global states of cellular automata with infinite geometries can only be given finite descriptions if we impose a condition that all but finitely many cells have the same state - which need not be explicitly represented. This is similar to the requirement that all but finitely many spaces on the infinite tape of a Turing machine remain blank, or the prohibition on infinite terms in the lambda calculus.

\section{Cellular spaces with Dynamics}

We now have all the necessary components of a general theory of cellular automata as labeled abstract cellular complexes. In this section, we will see how the geometric and dynamic components work together, and I'll use this theory to describe two specific, relatively well-known CA systems. While the conceptual technology involved can be employed in other ways, here we'll imagine dots as sites of atomic computation and links, tiles, and blocks as as information channels and supportive geometric infrastructure.

Definition. A cellular automaton consists of a nonempty set of AC complexes called geometries, a family of labeling functions from the geometry's cells onto a single finite set of states, and a single finite rule function that takes a specific closed labeled subcomplex in the geometry to an open labeled subcomplex having the same boundary. Applying the rule to each of its domains produces a new labeled geometry.

A rule for a geometry of dimension $d$ may thus be interpreted as a disjoint collection of $(d+1)$-dimensional labeled AC complexes called rule cylinders, one for each distict labeling of the rule's geometric domain. A rule cylinder consists of a labeled domain complex, a separate and corresponding labeled range complex, directed links from the dots of the former to the dots of the latter, and any higher dimensional cells that those links may bound. A history is an extended labeled geometry produced by recursively gluing the appropriate rule cylinders to all geometric subcomplexes with cell labels matching the rule cylinder domain: the labeled cells in the rule cylinder range constitute the geometry of the CA's next computational 'step'.

4.1. Wolfram's rule 110. A particularly well-publicized family of one-dimensional cellular automata, ambitiously named elementary CA, have the geometry of the integers - a connected infinite uniform 1-complex where every dot has a smallest neighborhood consisting of two links. Indeed, their geometry preserves the order of the integers as well, in that each dot knows its left from its right link. An elementary CA rule is defined on a domain consisting of the labelings of the smallest closed neighborhood of a dot $o, \sigma(o) \cup \beta(\sigma(o))$, 
and has as its range the labelings of $\sigma(o)$. Apart from their implicit orientation, geometric links remain unlabeled, and most descriptions of elementary CA don't even acknowledge their existence. The dots are labeled with a single binary digit, which I've expressed in the figures as black for 1 and white for 0 . The three dots in the domain complex together have $2^{3}=8$ possible labelings. An elementary CA rule must assign a one-bit label to each of these domain states: there are $2^{8}=256$ distinct ways to do that and thus 256 elementary CA rules, although identifying left-right and 0-1 symmetries reduces the functionally distinct rules to just 64 equivalence classes. These rules are conventionally named by arranging the eight labeled domains in descending numerical order and reading the corresponding range labels as a binary number. Stephen Wolfram, having discovered that some elementary CA rules exhibit chaotic dynamics [6], began an extensive empirical research program into cellular automata and related systems, culminating in a popular book [7]. In 2002 Matthew Cook proved that rule 110 can be given a state configuration (an initial labeling) capable of emulating a Turing machine, a construction which in 2009 he improved to compute in polynomial time [2].

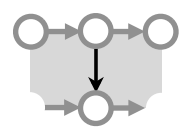

(A) Rule cylinder

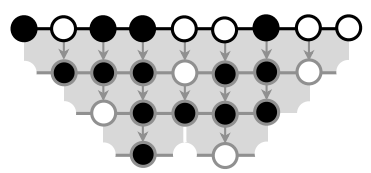

(в) Rule 110 history

FigURE 4. Structure of an elementary cellular automaton

Figure 4a shows one of rule 110's eight rule cylinders, including the orientation of the horizontal geometric links, which I'll leave implicit in subsequent figures. The horizontal arrows do not represent the direction of information flow, but merely distinguish left from right relative to the central dot. The arrow on the black vertical link doesn't represent information flow either, at least in terms of state. It is rather a structural identification between the upper and lower central dots, in the domain and range geometries respectively. The square tiles left and right are best thought of as together representing the information flow from the states of the domain dots to the state of the range dot. Figure $4 \mathrm{~b}$ shows a rule 110 history composed from multiple rule cylinders applied to the initial states in the top row of cells. The labeled dots of each row, or 'time step,' can be thought of as constructed simultaneously, or in any sequential order: Although the tiles and links in the cylinders domains overlap, they don't bear any state, so the result is the same regardless of rule application order and the system does not require global synchronization. It should be clear that this construction can be extended indefinitely downward, or 'forward in time,' so long as the initial row of cells extends infinitely left and right, and that the state configurations of this history depend only on the configuration of initial dot states, all but finitely many of which may be assumed to be 0's. Information spreads downward through the history at a maximum rate of one horizontal (or 'spatial') link per vertical ('temporal') link: this is sometimes called the 'speed of light,' and the ziggurat-shaped subcomplexes delineated by 
tracing state information forward or backward through the history are accordingly called 'light cones.'

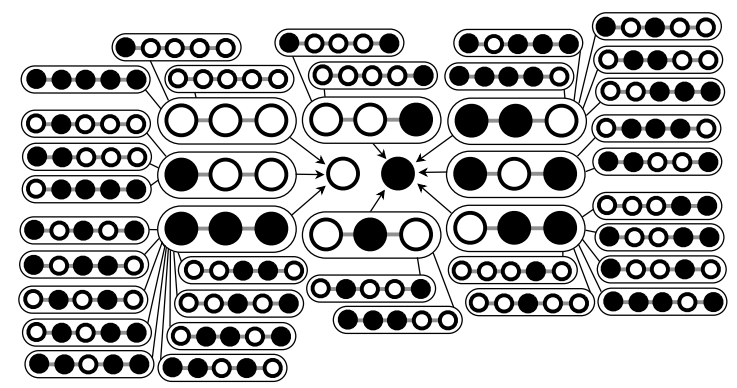

FiguRE 5. Rule 110 extended

A history complex can be a very useful view of a cellular automaton, but it's not the only view. While a history is limited by a particular initial state configuration, sometimes we may be interested in all possible CA states. In these cases, we can use another labelled cell complex representation - that of a state transition system. Here the 0-cells are labelled by states of connected subcomplexes, and the directed links represent combined rule applications constrained only by available information. Figure 5 shows all the ways in which either a 0 or a 1 can be produced by any combination of up to five contiguous dot states: in other words, all possible two-step backward light cones. The inner ring of eight three-dot states depicts what is usually thought of as the rule itself, while the outer clusters of five-dot states show all the possible ways to produce the three-dot states. This graph can be extended indefinitely, although the number of subcomplex state labels grows exponentially with each backward step. Still, this global state-space view can be insightful: already we can see that the sequence 111 follows from nine different five-cell states, while 101 is only reached from two of these. If we were looking for Eden states, this is how we might find them.

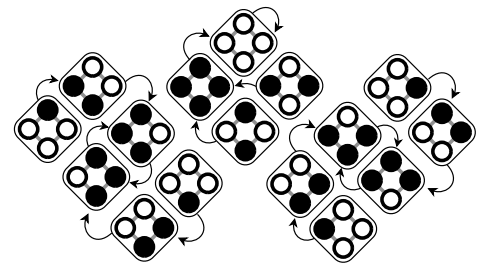

(A) A four-dot geometry

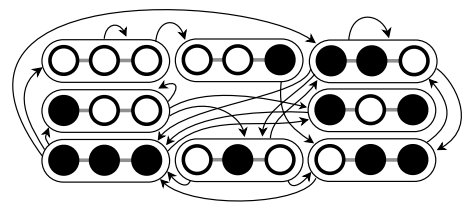

(B) A three-dot region

FiguRE 6. Rule 110 as a finite automaton

If we want to wrap up the infinitely branching deterministic structure of Figure 5 into a finite package there are two different basic approaches, as illustrated in Figure 6 . First, we could render the global geometry itself finite by wrapping it into a cycle: Figure 6a shows 
the transitions between all sixteen possible labelings of a four-dot geometry. Note that all four states with only one black dot are Eden states. Furthermore, the finite state space graph is partitioned into three disjoint subgraphs called 'attractor basins,' of which two are rotations of each other. This might be expected, since CA rules are coordinate-free and thus invariant under cyclic translations - in surveying bigger finite state-spaces, it can be helpful to form equivalence classes. This is the approach to finite spaces pioneered by Andrew Wuensche [8].

Alternatively, we could look at all possible transitions in a finite geometric subcomplex: Figure $6 \mathrm{~b}$ is an example. In elementary $\mathrm{CA}$, there are at most four of these transitions for any connected region, regardless of extent, since there are always only two dots on the region's boundary. Although I haven't done so in the figure, one could label the transition by the boundary states they represent. This kind of automaton graph is self-contained but non-deterministic: it shows exhaustively which temporal sequences are possible in a subcomplex subject to unknown boundary states, and has the nice property of being composable from - and decomposable into — smaller complete graphs for smaller subcomplexes.

4.2. Conway's Game of Life. The most well-known cellular automaton was invented by John H. Conway as a 'zero-player game' in 1970. Popularized by Martin Gardner, it was soon implemented in computers far and wide, and enjoys a devoted following to this day. Its simple rule can support a remarkably diverse range of complex structures, including several which have been shown to be Turing-complete.

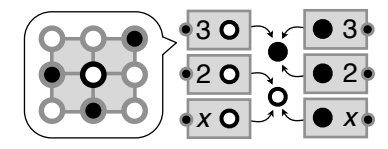

(A) Rule and domain: $x<2$ or $3<x$

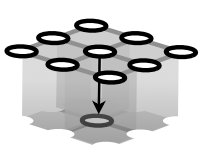

(в) Rule cylinder

Figure 7. Conway's Life CA

Life's geometry is a uniform tiling complex with square tiles, and its dots have one of two states. The domain of its rule, shown in Figure 7a, is the closure of the smallest neighborhood of a dot. The rules treats the eight boundary dots of the domain separately from the central dot: it just counts those with black (or 'live') state, regardless of their position on the boundary. If the central dot is white (or 'dead'), and there are exactly three black boundary dots, the rule labels the central dot black in the next step. If, on the other hand, the central dot is already black, then it will turn white unless there are either two or three black boundary cells. The Life rule thereby collapses the $2^{9}=512$ different domain states into just four equivalence classes of different sizes, although I've shown six in the figure for symmetry. These have the colorful names of 'birth,' 'death,' 'survival,' and 'stasis.' The white-to-white stasis condition is rarely mentioned, under the assumption that all but finitely many dots are labeled white at any step in the CA's evolution: an efficient computer implementation can ignore white cells altogether. 
Since the rule is insensitive to rotation, the geometry need not be oriented - a Life dot can't tell its north from its west neighbor, nor southeast from northwest. Consequently, we don't really need 512 rule cylinders, and may be able to use as few as 4, depending on how we think about matching the labeled dots in the rule domain. In any case, the AC complex structure of a rule cylinder is shown in Figure 7b: it includes four blocks, four tiles, and one directed link between the domain and range geometry subcomplexes. Just like the one-dimensional elementary $\mathrm{CA}$, these rule cylinders can be applied simultaneously or in any sequential order to construct a new labeled geometry from an existing one. The three-dimensional histories thereby produced are not as readable as the flat histories of one-dimensional CA, but on the other hand they're still easier to visualize than the four-dimensional histories of three-dimensional CA, which could be built using the same topological apparatus.

\section{Conclusion}

Cellular automata are a spatially distributed model of parallel computation. While nearly as old as the Turing machine, and with a good track record as a basis for scientific models in fields from physics to sociology, they have seen limited use in engineering and are often still regarded as 'exotic' or 'nonstandard' forms of computation. Nonetheless CA have much to teach us, as we increasingly understand our world in computational terms and continue to build larger and more widely distributed computing systems. Unfortunately, the many diverse varieties of CA are typically characterized in an ad-hoc fashion that obscures the underlying unity of their digital geometric structure and localized state transitions. Worse, these ad-hoc descriptions typically make unrestricted use of global coordinate systems for alignment and synchronization, and thus fail to highlight the most important features of this intrinsically decentralized theory of information processing.

Meanwhile, the rigorously defined and highly flexible conceptual technology of topological cell complexes, which was invented nearly 150 years ago in order to compute certain otherwise uncomputable properties of uncountable point-set spaces, and which has been the very basis for the highly successful modern algebraic approach to topology, remains all but unknown outside the topology research community. Cell complexes can be given sound geometric interpretations, and are closely related to the graph-based formalisms, such as labeled transition systems, that form the theoretical basis for modern programming languages. They have all the right topological and computational properties for a general theory of cellular automata. In this paper, I have shown how such a theory could proceed.

Beginning with ZF set theory, we have progressively refined the notion of a locally finite space into the digital geometric structure of an abstract cell complex, while proving that these structures are indeed T0 topologies in the classical sense, equipped with workable notions of boundary and connectedness. We have seen how an infinite AC complex can serve as the geometric structure of a cellular automaton, and how it can be labeled with states and given transition rules which determine its dynamics without any form of global coordinate system or nonlocal information transfer. Finally, I have shown two concrete applications of the theory to definitions of two well-known cellular automata. 


\section{REFERENCES}

[1] Gardner, M. (October 1970). "The fantastic combinations of John Conway's new solitaire game 'life'." Scientific American 223. pp. 120123.

[2] Cook, M. "A Concrete View of Rule 110 Computation." in T. Neary, D. Woods, A.K. Seda and N. Murphy (eds.): The Complexity of Simple Programs 2008, pp. 3155. doi:10.4204/EPTCS.1.4 http://arxiv.org/abs/0906.3248

[3] Kovalevsky, V. (2006) "Axiomatic Digital Topology." Journal of Mathematical Imaging and Vision 26: 4158. doi:10.1007/s10851-006-7453-6

[4] Klette, R. and Rosenfeld, A. Digital Geometry: Geometric Methods for Digital Picture Analysis. Morgan Kauffman Publishers, 2004. ISBN:1-55860-861-3

[5] Rendell, P. (2000) A Turing Machine implemented in Conway's Game of Life. http://rendell-attic.org/gol/tm.htm

[6] Wolfram, S. (1984). "Computation theory of cellular automata". Communications in Mathematical Physics 96: 1557. doi:10.1007/BF01217347

[7] Wolfram, S. A New Kind of Science. Wolfram Media, Inc., 2002. ISBN:1-57955-008-8

[8] Wuensche, A. and Lesser, M.J. The Global Dynamics of Cellular Automata. Addison-Wesley, 1992. ISBN: 0-201-55740-1 